\title{
Some Edge Product Cordial Graphs in the Context of Duplication of Some Graph Elements
}

\author{
Udayan M. Prajapati' ${ }^{1}$, Prakruti D. Shah ${ }^{2}$ \\ ${ }^{1}$ St. Xavier's College, Ahmedabad, India \\ ${ }^{2}$ Shankersinh Vaghela Bapu Institute of Technology, Gandhinagar, India \\ Email: udayan64@yahoo.com,prakrutishah29@gmail.com
}

How to cite this paper: Prajapati, U.M. and Shah, P.D. (2016) Some Edge Product Cordial Graphs in the Context of Duplication of Some Graph Elements. Open Journal ot Discrete Mathematics, 6, 248-258.

http://dx.doi.org/10.4236/ojdm.2016.64021

Received: June 8, 2016

Accepted: September 6, 2016

Published: September 9, 2016

Copyright $(\odot 2016$ by authors and Scientific Research Publishing Inc. This work is licensed under the Creative Commons Attribution International License (CC BY 4.0).

http://creativecommons.org/licenses/by/4.0/

\begin{abstract}
For a graph $G=(V(G), E(G))$, a function $f: E(G) \rightarrow\{0,1\}$ is called an edge product cordial labeling of $G$, if the induced vertex labeling function is defined by the product of the labels of the incident edges as such that the number of edges with label 1 and the number of edges with label 0 differ by at most 1 and the number of vertices with label 1 and the number of vertices with label 0 differ by at most 1 . In this paper, we show that the graphs obtained by duplication of a vertex, duplication of a vertex by an edge or duplication of an edge by a vertex in a crown graph are edge product cordial. Moreover, we show that the graph obtained by duplication of each of the vertices of degree three by an edge in a gear graph is edge product cordial. We also show that the graph obtained by duplication of each of the pendent vertices by a new vertex in a helm graph is edge product cordial.
\end{abstract}

\section{Keywords}

Graph Labeling, Edge Product Cordial Labeling, Duplication of a Vertex

\section{Introduction}

We begin with a simple, finite, undirected graph $G=(V(G), E(G))$ where $V(G)$ and $E(G)$ denote the vertex set and the edge set respectively. For all other terminology, we follow Gross [1]. We will provide a brief summary of definitions and other information which are necessary for the present investigations.

Definition 1. A graph labeling is an assignment of integers to the vertices or edges or both subject to certain condition. If the domain of the mapping is the set of vertices, edges or both then the labeling is called a vertex labeling, an edge labeling or a total labeling. 
Definition 2. For a graph $G$, an edge labeling function is defined as $f: E(G) \rightarrow\{0,1\}$ and the induced vertex labeling function $f^{*}: V(G) \rightarrow\{0,1\}$ is given by $f^{*}(v)=f\left(e_{1}\right) f\left(e_{2}\right) \cdots f\left(e_{k}\right)$ if $e_{1}, e_{2}, \cdots, e_{k}$ are the edges incident with the vertex $v$.

We denote the number of vertices of $G$ having label $i$ under $f^{*}$ by $v_{f}(i)$ and the number of edges of $G$ having label $i$ under $f$ by $e_{f}(i)$ for $i=0,1$.

The function $f$ is called an edge product cordial labeling of $G$ if $\left|v_{f}(0)-v_{f}(1)\right| \leq 1$ and $\left|e_{f}(0)-e_{f}(1)\right| \leq 1$. A graph $G$ is called edge product cordial if it admits edge product cordial labeling.

The concept of edge product cordial labeling was introduce by Vaidya and Barasara [2] in which they proved that $C_{n}$ for $n$ odd, trees of order greater than 2, unicyclic graphs of odd order, crowns, armed crowns, helms, closed helms, webs, flowers graph are edge product cordial. They also proved that wheel and gear for even are not edge product cordial. They also [3] proved that $T_{n}, D T_{n}$ for odd, $Q_{n}$ for odd, $D Q_{n}$ for odd are edge product cordial labeling. They also proved that $D T_{n}$ for even, $Q_{n}$ for even, $D Q_{n}$ for even, $D F_{n}$ are not edge product cordial labeling.

Definition 3. The graph $W_{n}=C_{n}+K_{1}$ is called wheel graph, the vertex corresponding to $K_{1}$ is called apex vertex and vertices corresponding to $C_{n}$ are called rim vertices.

Definition 4. The helm $H_{n}$ is the graph obtained from a wheel $W_{n}$ by attaching a pendent edge at each vertex of the n-cycle.

Definition 5. A gear graph is obtained from the wheel graph $W_{n}$ by adding a vertex between every pair of adjacent vertices of the n-cycle.

Definition 6. The crown $C_{n} \odot K_{1}$ is obtained by joining a single pendent edge to each vertex of $C_{n}$.

Definition 7. The neighborhood of a vertex $v$ of a graph is the set of all vertices adjacent to $\mathrm{v}$. It is denoted by $N(\mathrm{v})$.

Definition 8. Duplication of a vertex of the graph $G$ is the graph $G^{\prime}$ obtained from $G$ by adding a new vertex $v^{\prime}$ to $G$ such that $N\left(v^{\prime}\right)=N(v)$.

Definition 9. Duplication of a vertex $v_{k}$ by a new edge $e=v_{k}^{\prime} v_{k}^{\prime \prime}$ in a graph $G$ produces a new graph $G^{\prime}$ such that $N\left(v_{k}^{\prime}\right)=\left\{v_{k}, v_{k}^{\prime \prime}\right\}$ and $N\left(v_{k}^{\prime \prime}\right)=\left\{v_{k}, v_{k}^{\prime}\right\}$.

The concept of duplication of vertex by edge was introduce by Vaidya and Barasara [4].

Definition 10. Duplication of an edge $e=u v$ by a new vertex $w$ in a graph $G$ produces a new graph $G^{\prime}$ such that $N(w)=\{u, v\}$.

The concept of duplication of edge by vertex was introduce by Vaidya and Dani [5].

\section{Main Results}

Theorem 1. The graph obtained by duplication of an arbitrary vertex of the cycle in a crown graph is an edge product cordial graph.

Proof. Let $C_{n}$ be a cycle with consecutive vertices $v_{1}, v_{2}, \cdots, v_{n}$ and edges $e_{i}=v_{i} v_{i+1}$ for each $i \in\{1,2,3, \cdots, n\}$. Let $u_{i}$ be a new vertex adjacent to $v_{i}$ with $e_{i}^{\prime}=u_{i} v_{i}$ for each $i \in\{1,2,3, \cdots, n\}$. Resulting graph is a crown graph $G_{1}$. 
Let $G$ be the graph obtained by duplication of the vertex $v_{n}$ by a new vertex $v^{\prime}$ of $G_{1}$ such that $e_{1}^{\prime \prime}=v^{\prime} v_{1}, e_{2}^{\prime \prime}=v^{\prime} v_{n-1}$ and $e_{3}^{\prime \prime}=v^{\prime} u_{n}$.

Thus $|V(G)|=2 n+1$ and $|E(G)|=2 n+3$.

Now for $n=3$ and $n=4$ Figure 1 shows that the graphs are edge product cordial as follows:
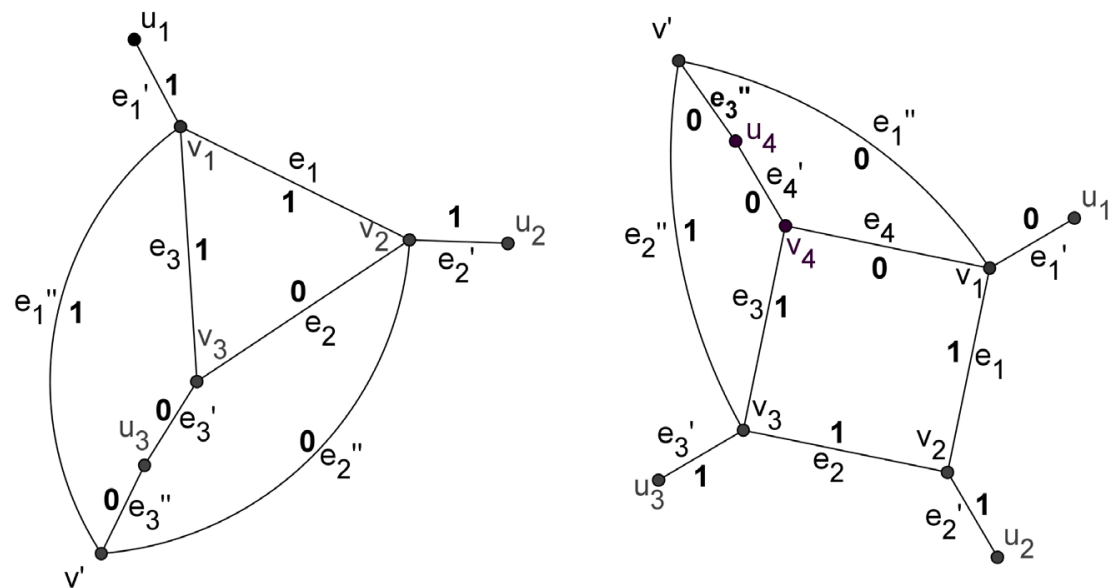

Figure 1. $n=3$ and $n=4$.

Case 1: When $n$ is odd. For $n \geq 5$, define $f: E(G) \rightarrow\{0,1\}$ as follows:

$$
f(e)=\left\{\begin{array}{l}
1, \text { if } e=e_{i}, \text { for } 1 \leq i \leq\left\lceil\frac{n}{2}\right\rceil ; \\
0, \text { if } e=e_{i}, \text { for }\left\lceil\frac{n}{2}\right\rceil+1 \leq i \leq n ; \\
1, \text { if } e=e_{i}^{\prime}, \text { for } 1 \leq i \leq\left\lceil\frac{n}{2}\right\rceil ; \\
0, \text { if } e=e_{i}^{\prime}, \text { for }\left\lceil\frac{n}{2}\right\rceil+1 \leq i \leq n ; \\
0, \text { if } e=e_{i}^{\prime \prime}, \text { for } i=1,2,3 .
\end{array}\right.
$$

In the view of above labeling pattern we have,

$$
v_{f}(1)=v_{f}(0)-1=n \text { and } e_{f}(1)=e_{f}(0)-1=n+1 .
$$

Case 2: When $n$ is even. For $n \geq 6$, define $f: E(G) \rightarrow\{0,1\}$ as follows:

$$
f(e)=\left\{\begin{array}{l}
1, \text { if } e=e_{i}, \text { for } 1 \leq i \leq \frac{n}{2}+1 ; \\
0, \text { if } e=e_{i}, \text { for } \frac{n}{2}+2 \leq i \leq n ; \\
1, \text { if } e=e_{i}^{\prime}, \text { for } 1 \leq i \leq \frac{n}{2}+1 ; \\
0, \text { if } e=e_{i}^{\prime}, \text { for } \frac{n}{2}+2 \leq i \leq n ; \\
0, \text { if } e=e_{i}^{\prime \prime}, \text { for } i=1,2,3 .
\end{array}\right.
$$


In the view of the above labeling pattern we have,

$$
v_{f}(0)=v_{f}(1)-1=n \text { and } e_{f}(0)=e_{f}(1)-1=n+1 .
$$

Thus, from both the cases we have $\left|v_{f}(0)-v_{f}(1)\right| \leq 1$ and $\left|e_{f}(0)-e_{f}(1)\right| \leq 1$.

Hence, graph $G$ admits edge product cordial labeling. Thus, $G$ is an edge product cordial graph.

Illustration 1. The graph obtained by duplication of an arbitrary vertex of the cycle $C_{n}$ in a crown graph is an edge product cordial graph as shown in Figure 2 as follows.
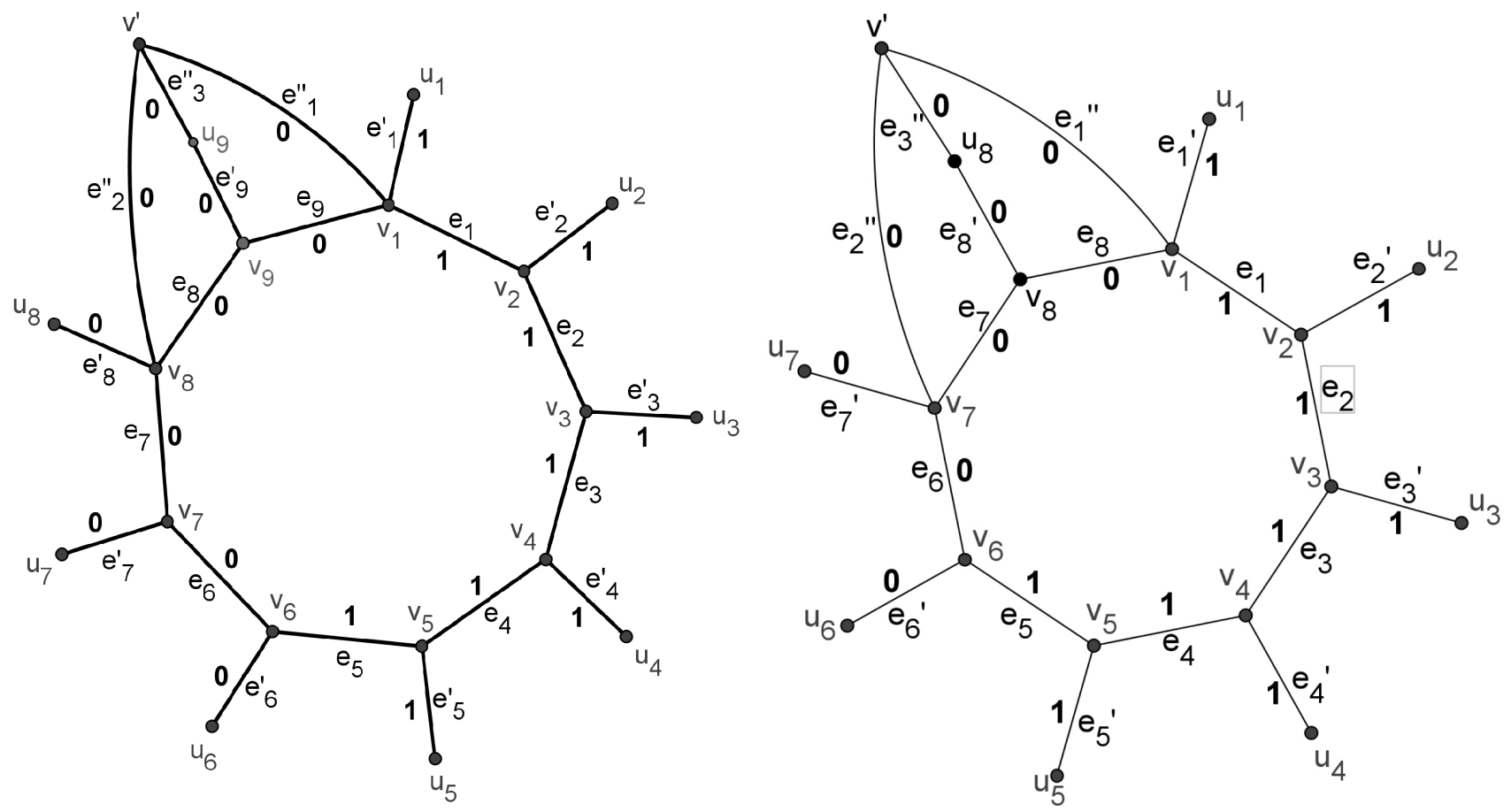

Figure 2. $n=9$ and $n=8$.

Theorem 2. The graph obtained by duplication of an arbitrary vertex of the cycle by a new edge in a crown graph is edge product cordial graph.

Proof. Let $C_{n}$ be a cycle with consecutive vertices $v_{1}, v_{2}, \cdots, v_{n}$ and edges $e_{i}=v_{i} v_{i+1}$ for each $i \in\{1,2, \cdots, n\}$. Let $u_{i}$ be a new vertex adjacent to $v_{i}$ with $e_{i}=u_{i} v_{i}$ for each $i \in\{1,2, \cdots, n\}$. Resulting graph is a crown graph $G_{1}$.

Let $G$ be the graph obtained by duplication of the vertex $v_{n}$ by an edge $e_{1}^{\prime \prime}=v^{\prime} v^{\prime \prime}$ of $G_{1}$ such that $e_{2}^{\prime \prime}=v^{\prime} v_{n}$ and $e_{3}^{\prime \prime}=v^{\prime \prime} v_{n}$. Thus $|V(G)|=2 n+2$ and $|E(G)|=2 n+3$. Define $f: E(G) \rightarrow\{0,1\}$ as follows:

$$
f(e)=\left\{\begin{array}{l}
1, \text { if } e=e_{i}, \text { for } i=1,2 \\
0, \text { if } e=e_{i}, \text { for } 3 \leq i \leq n \\
1, \text { if } e=e_{i}^{\prime} \text {, for } i \in\{1,2, \cdots, n\} \\
0, \text { if } e=e_{i}^{\prime \prime}, \text { for } i=1,2,3 .
\end{array}\right.
$$

In the view of the above labeling pattern we have, $v_{f}(0)=v_{f}(1)=n+1$ and 
$e_{f}(0)=e_{f}(1)-1=n+1$. Thus, we have $\left|v_{f}(0)-v_{f}(1)\right| \leq 1$ and $\left|e_{f}(0)-e_{f}(1)\right| \leq 1$.

Hence, graph $G$ admits edge product cordial labeling. Thus, $G$ is an edge product cordial graph.

Illustration 2. The graph obtained by duplication of an arbitrary vertex of the cycle $C_{n}$ by a new edge in a crown graph is edge product cordial graph as shown in Figure 3.

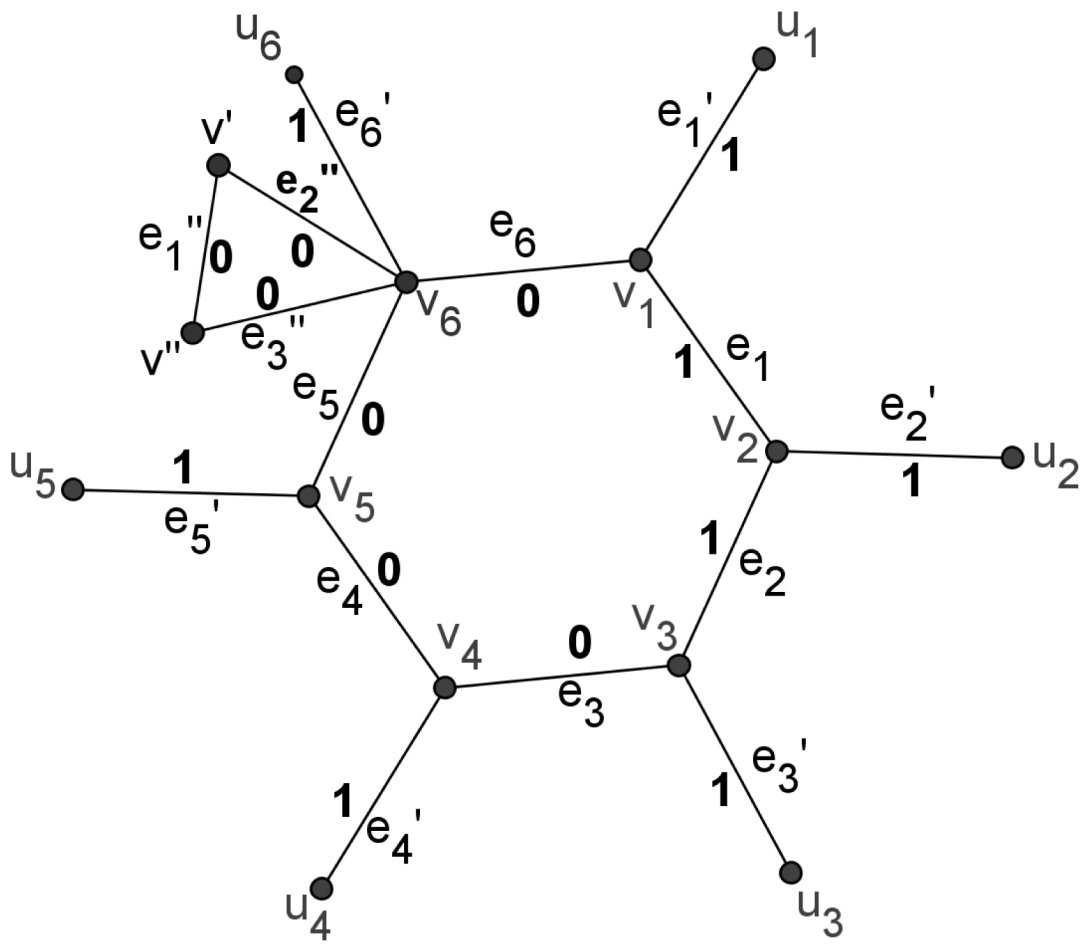

Figure 3. $n=6$.

Theorem 3. The graph obtained by duplication of an arbitrary edge of the cycle $C_{n}$ by a new vertex in a crown graph is edge product cordial.

Proof. Let $C_{n}$ be a cycle with the consecutive vertices $v_{1}, v_{2}, \cdots, v_{n}$ and edges $e_{i}=v_{i} v_{i+1}$ for each $i \in\{1,2, \cdots, n\}$. Let $u_{i}$ be a new vertex adjacent to $v_{i}$ with $e_{i}^{\prime}=u_{i} v_{i}$ for each $i \in\{1,2, \cdots, n\}$. Resulting graph is a crown graph $G_{1}$.

Let $G$ be the graph obtained by duplication of an edge $e_{1}=v_{1} v_{2}$ by a vertex $v^{\prime}$ in $G_{1}$ such that $e_{1}^{\prime \prime}=v^{\prime} v_{1}$ and $e_{2}^{\prime \prime}=v^{\prime} v_{2}$. Thus $|V(G)|=2 n+1$ and $|E(G)|=2 n+2$. Define $f: E(G) \rightarrow\{0,1\}$ as follows:

$$
f(e)=\left\{\begin{array}{l}
1, \text { if } e=e_{i}, \text { for } i=1 \\
0, \text { if } e=e_{i}, \text { for } 2 \leq i \leq n ; \\
1, \text { if } e=e_{i}^{\prime}, \text { for } i \in\{1,2, \cdots, n\} ; \\
0, \text { if } e=e_{i}^{\prime \prime}, \text { for } i=1,2 .
\end{array}\right.
$$

In the view of above labeling pattern we have, $v_{f}(0)-1=v_{f}(1)=n$ and $e_{f}(0)=e_{f}(1)=n+1$. Thus, we have $\left|v_{f}(0)-v_{f}(1)\right| \leq 1$ and $\left|e_{f}(0)-e_{f}(1)\right| \leq 1$.

Hence, graph $G$ admits edge product cordial labeling. Thus, $G$ is an edge product 
cordial graph.

Illustration 3. The graph obtained by duplication of an arbitrary edge of the cycle $C_{n}$ by a new vertex in a crown graph is edge product cordial graph as shown in Figure 4.

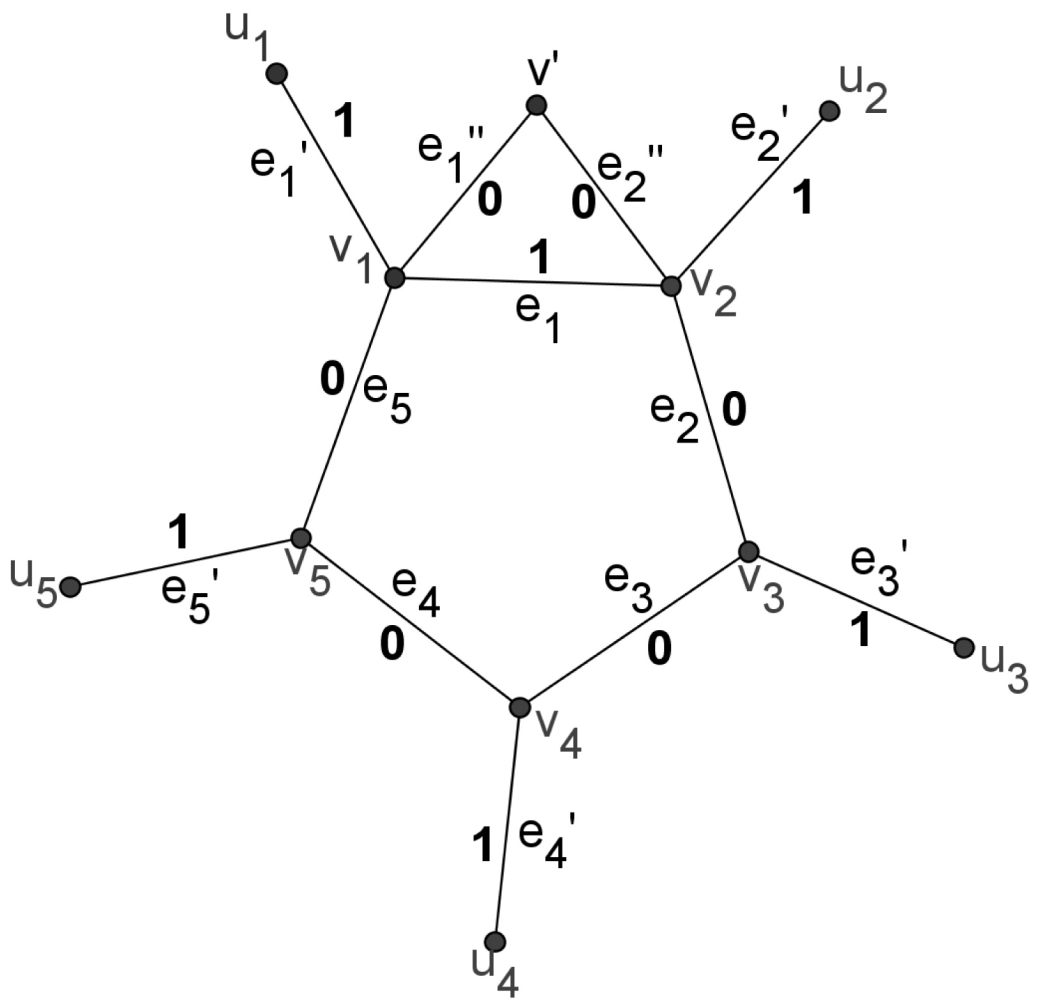

Figure 4. $n=5$.

Theorem 4. The graph obtained by duplication of each pendent vertex by a new vertex in a crown graph is edge product cordial graph.

Proof. Let $C_{n}$ be a cycle with consecutive vertices $v_{1}, v_{2}, \cdots, v_{n}$ and edges $e_{i}=v_{i} v_{i+1}$ for each $i \in\{1,2, \cdots, n\}$. Let $u_{i}$ be a new vertex adjacent to $v_{i}$ with $e_{i}^{\prime}=u_{i} v_{i}$ for each $i \in\{1,2, \cdots, n\}$. Resulting graph is a crown graph $G_{1}$.

Let $G$ be the graph obtained by duplication of each pendent vertex $u_{i}$ by a new vertex $v_{i}^{\prime}$ of $G_{1}$ such that $e_{i}^{\prime \prime}=v_{i}^{\prime} v_{i}$ for $i \in\{1,2, \cdots, n\}$. Thus $|V(G)|=3 n$ and $|E(G)|=3 n$.

Case 1: When $n$ is odd, define $f: E(G) \rightarrow\{0,1\}$ as follows:

$$
f(e)=\left\{\begin{array}{l}
0, \text { if } e=e_{i}, \text { for } i \in\{1,2, \cdots, n\} ; \\
1, \text { if } e=e_{i}^{\prime}, \text { for } i \in\{1,2, \cdots, n\} ; \\
1, \text { if } e=e_{i}^{\prime \prime}, 1 \leq i \leq\left\lceil\frac{n}{2}\right\rceil ; \\
0, \text { if } e=e_{i}^{\prime \prime},\left\lceil\frac{n}{2}\right\rceil+1 \leq i \leq n .
\end{array}\right.
$$

In the view of above labeling pattern we have, 


$$
v_{f}(0)=v_{f}(1)-1=\left\lfloor\frac{3 n}{2}\right\rfloor \text { and } e_{f}(0)=e_{f}(1)-1=\left\lfloor\frac{3 n}{2}\right\rfloor .
$$

Case 2: When $n$ is even, define $f: E(G) \rightarrow\{0,1\}$ as follows:

$$
f(e)=\left\{\begin{array}{l}
0, \text { if } e=e_{i}, \text { for } i \in\{1,2, \cdots, n\} ; \\
1, \text { if } e=e_{i}^{\prime}, \text { for } i \in\{1,2, \cdots, n\} ; \\
1, \text { if } e=e_{i}^{\prime \prime}, 1 \leq i \leq \frac{n}{2} \\
0, \text { if } e=e_{i}^{\prime \prime}, \frac{n}{2}+1 \leq i \leq n .
\end{array}\right.
$$

In the view of above labeling pattern we have, $v_{f}(0)=v_{f}(1)=\frac{3 n}{2}$ and $e_{f}(0)=e_{f}(1)=\frac{3 n}{2}$.

Thus, from both the cases we have $\left|v_{f}(0)-v_{f}(1)\right| \leq 1$ and $\left|e_{f}(0)-e_{f}(1)\right| \leq 1$. Hence, graph $G$ admits edge product cordial labeling. Thus, $G$ is an edge product cordial graph.

Illustration 4. The graph obtained by duplication of each pendent vertex by a new vertex in a crown graph is edge product cordial graph as shown in Figure 5 as follows.
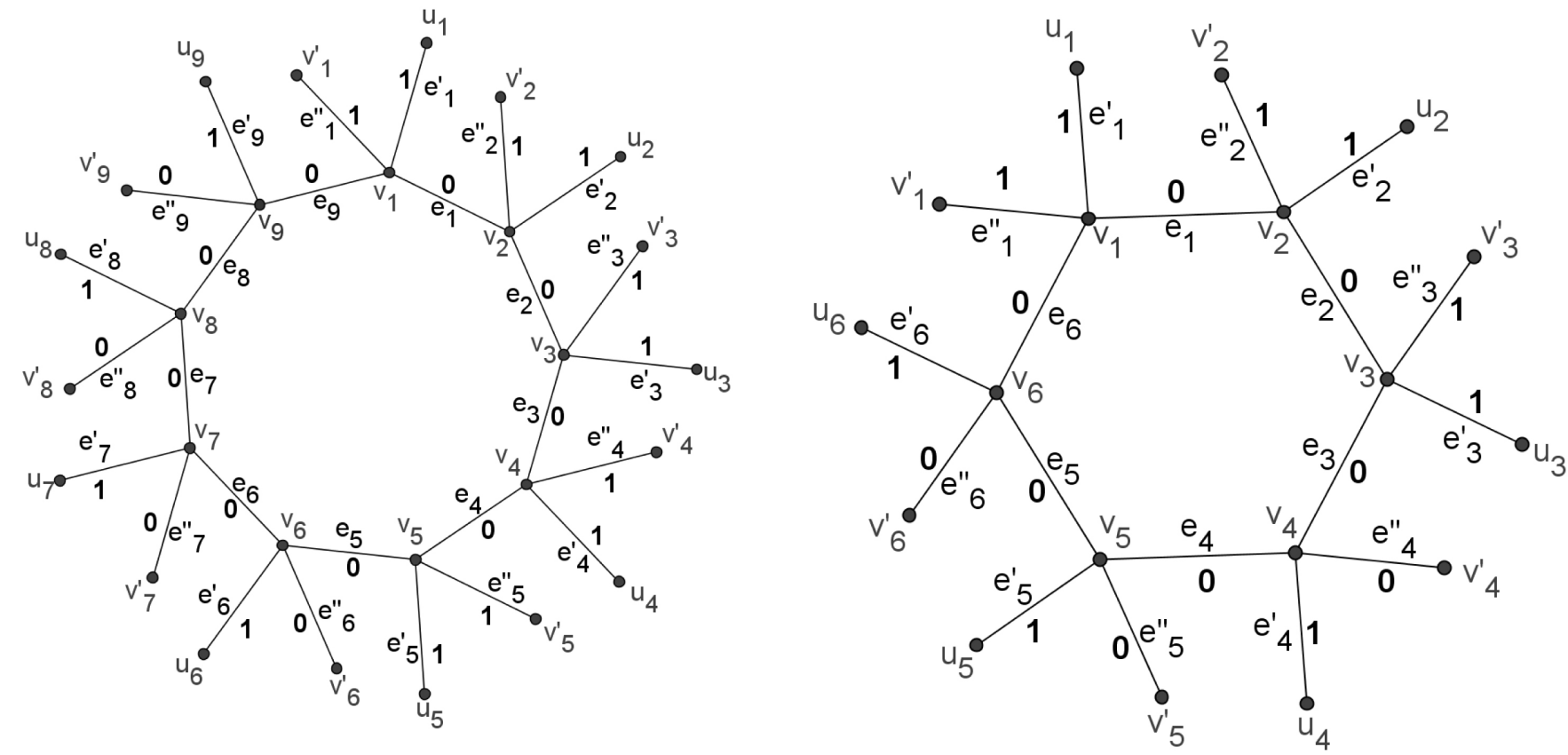

Figure 5. $n=9$ and $n=6$.

Theorem 5. The graph obtained by duplication of each of the vertices of degree three by an edge in a gear graph is an edge product cordial graph.

Proof. Let $W_{n}$ be the wheel graph with apex vertex $v$ and consecutive rim vertices $v_{1}, v_{2}, \cdots, v_{n}$. To obtained the gear graph $G_{n}$ subdivide each of the rim edges 
$v_{1} v_{2}, v_{2} v_{3}, \cdots, v_{n-1} v_{n}, v_{n} v_{1}$ of the wheel graph by the vertices $u_{1}, u_{2}, \cdots, u_{n}$ respectively such that $e_{i}=v v_{i}, \quad e_{l_{i}}=v_{i} u_{i}$ and $e_{r_{i}}=u_{i} v_{i+1}$ for $i \in\{1,2, \cdots, n\}$.

Let $G$ be the graph obtained from $G_{n}$ by duplication of each vertex $v_{i}$ by an edge $f_{i}=v_{i}^{\prime} v_{i}^{\prime \prime}$ such that $f_{i}^{\prime}=v_{i}^{\prime} v_{i}$ and $f_{i}^{\prime \prime}=v_{i}^{\prime \prime} v_{i}$ for $i \in\{1,2, \cdots, n\}$. Thus $|V(G)|=4 n+1$ and $|E(G)|=6 n$.

Define $f: E(G) \rightarrow\{0,1\}$ as follows:

$$
f(e)=\left\{\begin{array}{l}
0, \text { if } e=e_{i}, \text { for } i=\{1,2,3, \cdots, n\} ; \\
0, \text { if } e=e_{l_{i}}, \text { for } i=\{1,2,3, \cdots, n\} \\
0, \text { if } e=e_{r_{i}}, \text { for } i \in\{1,2,3, \cdots, n\} \\
1, \text { if } e=f_{i}, \text { for } i=\{1,2,3, \cdots, n\} ; \\
1, \text { if } e=f_{i}^{\prime}, \text { for } i=\{1,2,3, \cdots, n\} ; \\
1, \text { if } e=f_{i}^{\prime \prime}, \text { for } i=\{1,2,3, \cdots, n\} .
\end{array}\right.
$$

In the view of the above labeling pattern we have, $v_{f}(0)-1=v_{f}(1)=2 n$ and $e_{f}(0)=e_{f}(1)=3 n$. Thus, we have $\left|v_{f}(0)-v_{f}(1)\right| \leq 1$ and $\left|e_{f}(0)-e_{f}(1)\right| \leq 1$. Hence, graph $G$ admits edge product cordial labeling. Thus, $G$ is edge product cordial graph.

Illustration 5. The graph obtained by duplication of each vertex of degree three by an edge in a gear graph is an edge product cordial graph as shown in Figure 6.

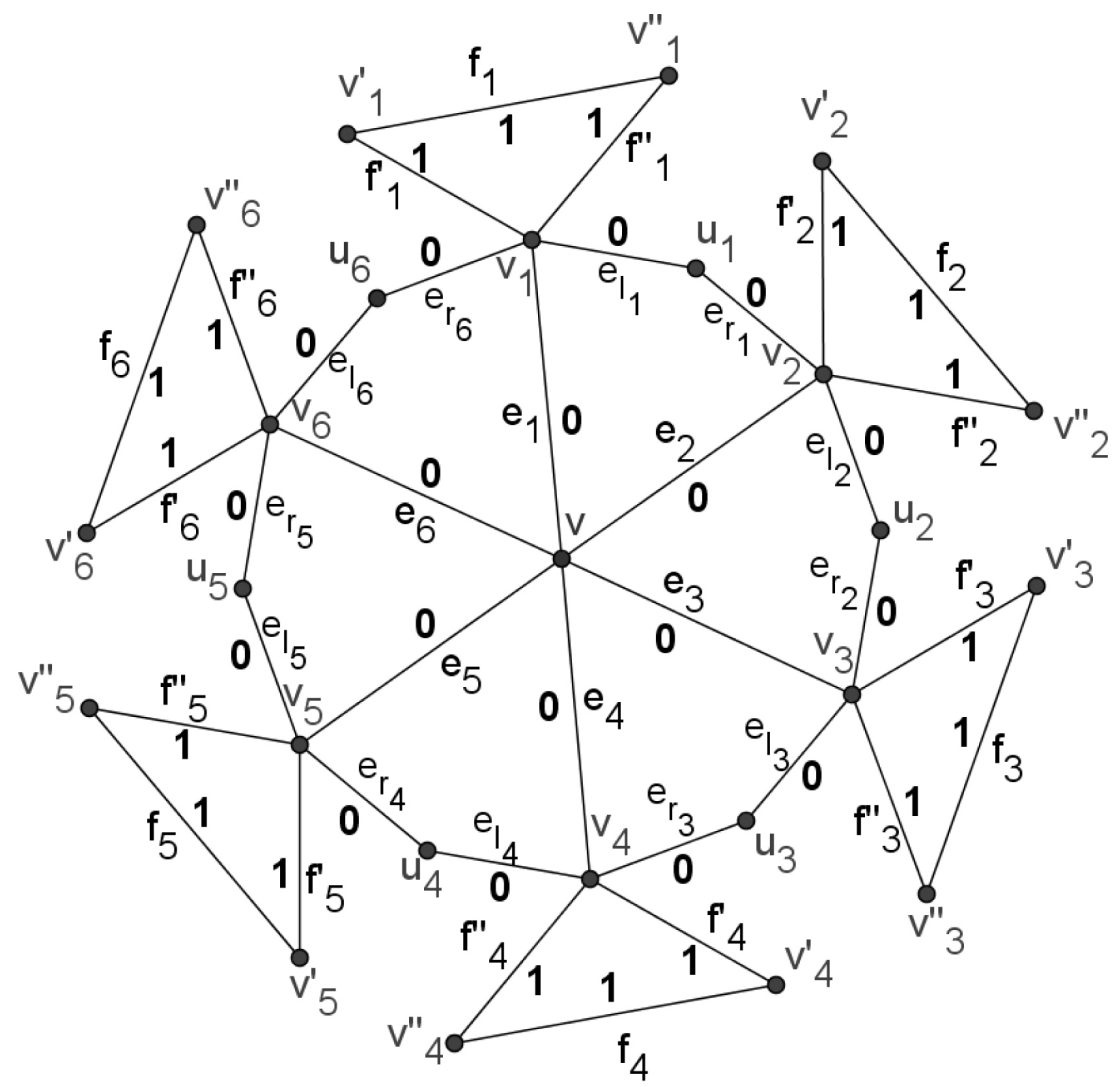

Figure 6. $n=6$. 
Theorem 6. The graph obtained by duplication of each of the pendent vertices by a new vertex in a helm graph is edge product cordial graph.

Proof. Let $v$ be the apex vertex and $v_{1}, v_{2}, \cdots, v_{n}$ be the consecutive rim vertices of the wheel $W_{n}$ with edges $e_{i}=v_{i} v_{i+1}$ and $e_{i}^{\prime}=v v_{i}$ for $i \in\{1,2, \cdots, n\}$. Let $u_{i}$ be a new vertex adjacent to $v_{i}$ with edges $e_{i}^{\prime \prime}=u_{i} v_{i}$ for $i \in\{1,2, \cdots, n\}$. Resulting graph is helm graph $H_{n}$.

Let $G$ be the graph obtained from $H_{n}$ by duplication of each pendent vertex $u_{i}$ by a new vertex $v_{i}^{\prime}$ such that $f_{i}=v_{i}^{\prime} v_{i}$ for $i \in\{1,2, \cdots, n\}$. Thus $|V(G)|=3 n+1$ and $|E(G)|=4 n$.

Case 1: When $n$ is odd, define $f: E(G) \rightarrow\{0,1\}$ as follows:

$$
f(e)=\left\{\begin{array}{l}
1, \text { if } e=e_{i}, 1 \leq i \leq\left\lfloor\frac{n}{2}\right\rfloor ; \\
0, \text { if } e=e_{i},\left\lfloor\frac{n}{2}\right\rfloor+1 \leq i \leq n ; \\
0, \text { if } e=e_{i}^{\prime} \text {, for } i \in\{1,2, \cdots, n\} ; \\
0, \text { if } e=e_{i}^{\prime \prime}, 1 \leq i \leq n-1 ; \\
1, \text { if } e=f_{i}^{\prime \prime}, 1 \leq i \leq\left\lfloor\frac{n}{2}\right\rfloor+2 ; \\
0, \text { if } e=f_{i},\left\lfloor\frac{n}{2}\right\rfloor+3 \leq i \leq n .
\end{array}\right.
$$

In the view of above labeling pattern we have, $v_{f}(0)=v_{f}(1)=\left\lceil\frac{3 n}{2}\right\rceil$ and $e_{f}(0)=e_{f}(1)=2 n$.

Case 2: When $n$ is even, define $f: E(G) \rightarrow\{0,1\}$ as follows:

$$
f(e)=\left\{\begin{array}{l}
1, \text { if } e=e_{i}, 1 \leq i \leq \frac{n}{2}-1 ; \\
0, \text { if } e=e_{i}, \frac{n}{2} \leq i \leq n ; \\
0, \text { if } e=e_{i}^{\prime}, \text { for } i \in\{1,2, \cdots, n\} ; \\
1, \text { if } e=e_{i}^{\prime \prime}, 1 \leq i \leq \frac{n}{2}+1 ; \\
0, \text { if } e=e_{i}^{\prime \prime}, \frac{n}{2}+2 \leq i \leq n ; \\
1, \text { if } e=f_{i}, \text { for } i \in\{1,2, \cdots, n\} .
\end{array}\right.
$$

In the view of above labeling pattern we have, $v_{f}(0)=v_{f}(1)-1=\frac{3 n}{2}$ and $e_{f}(0)=e_{f}(1)=2 n$.

Thus, from both the cases we have $\left|v_{f}(0)-v_{f}(1)\right| \leq 1$ and $\left|e_{f}(0)-e_{f}(1)\right| \leq 1$. Hence, graph $G$ admits edge product cordial labeling. Thus, $G$ is an edge product cordial graph. 
Illustration 6. The graph obtained by duplication of each pendent vertex by a new vertex in a helm graph is edge product cordial graph as shown in Figure 7.
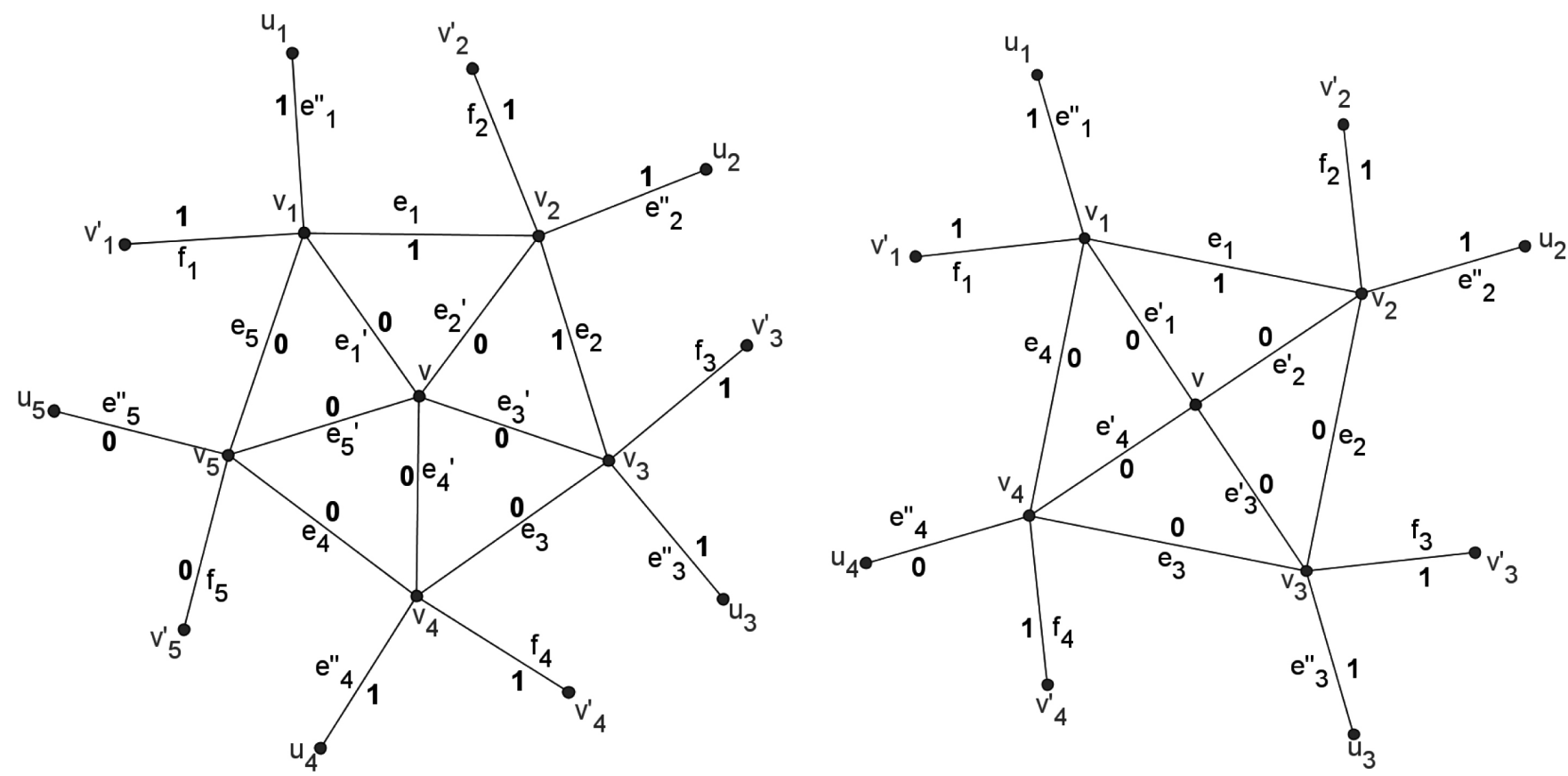

Figure 7. $n=5$ and $n=4$.

\section{Conclusion}

We have derived six results for edge product cordial related to crown graph, gear graph and helm graph in the context of duplication of various graph elements. Similar problem can be discussed for other graph family for edge product cordial labeling.

\section{Acknowledgements}

The authors are highly thankful to the anonymous referee for valuable comments and constructive suggestions. The First author is thankful to the University Grant Commission, India for supporting him with Minor Research Project under No. F. 47-903/ 14 (WRO) dated 11th March, 2015.

\section{References}

[1] Gross, J.L. and Yellen, J. (Eds.) (2004) Handbook of Graph Theory. CRC Press, Boca Raton.

[2] Vaidya, S.K. and Barasara, C.M. (2012) Edge Product Cordial Labeling of Graphs. Journal of Mathematics and Computer Science, 2, 1436-1450.

http://scik.org/index.php/jmcs/article/view/420/189

[3] Vaidya, S.K. and Barasara, C.M. (2013) Some New Families of Edge Product Cordial Graphs. Advanced Modeling Optimization, 15, 103-111. http://camo.ici.ro/journal/vol15/v15a9.pdf

[4] Vaidya, S.K. and Barasara, C.M. (2011) Product Cordial Graphs in the Context of Some Graph Operations. International Journal of Mathematics and Computer Science, 1, 1-6. 
[5] Vaidya, S.K. and Dani, N.A. (2011) Cordial and 3-Equitable Graphs Induced by Duplication of Edge. Mathematics Today, 27, 71-82.

Submit or recommend next manuscript to SCIRP and we will provide best service for you:

Accepting pre-submission inquiries through Email, Facebook, LinkedIn, Twitter, etc.

A wide selection of journals (inclusive of 9 subjects, more than 200 journals)

Providing 24-hour high-quality service

User-friendly online submission system

Fair and swift peer-review system

Efficient typesetting and proofreading procedure

Display of the result of downloads and visits, as well as the number of cited articles

Maximum dissemination of your research work

Submit your manuscript at: http://papersubmission.scirp.org/ 